\title{
Sharp contact corners, fretting and cracks
}

\author{
D. A. Hills, R. C. Flicek \\ Department of Engineering Science, University of Oxford, Parks Road, Oxford, OX1 3PJ, UK
}

D. Dini

Department of Mechanical Engineering, Imperial College London, South Kensington Campus, Exbibition Road, London, SW7 $2 A Z, U K$

\begin{abstract}
Contacts with sharp edges subject to oscillatory loading are likely to nucleate cracks from the corners, if the loading is sufficiently severe. To a first approximation, the corners behave like notches, where the local elastic behaviour is relieved by plasticity, and which in turn causes irreversibilities that give rise to crack nucleation, but also by frictional slip. One question we aim to answer here is; when is the frictional slip enveloped by plastic slip, so that the corner is effectively a notch in a monolithic material? We do this by employing the classical Williams asymptotic solution to model the contact corner, and, in doing so, we render the solution completely general in the sense that it is independent of the overall geometry of the components. We then re-define the independent parameters describing the properties of the Williams solution by using the inherent length scale, a procedure that was described at the first IJFatigue and FFEMS joint workshop [1]. By proceeding in this way, we can provide a self-contained solution that can be 'pasted in' to any complete contact problem, and hence the likelihood of crack nucleation, and the circumstances under which it might occur, can be classified. Further, this reformulation of Williams' solution provides a clear means of obtaining the strength (defined by crack nucleation conditions) of a material pair with a particular contact angle. This means that the results from a test carried out using a laboratory specimen may easily be carried over to any complicated contact problem found in engineering practice, and a mechanical test of the prototypical geometry, which may often be quite difficult, is avoided.
\end{abstract}

KEYWORDS. asymptotic approaches; complete contacts; fretting fatigue; mode mixity; sharp notches; small scale yielding; Williams solution

\section{INTRODUCTION}

$\mathrm{O}$ ur aim is to provide a framework for the understanding of fretting fatigue for complete contacts, when the geometry of the contact itself and both the type and history of loading are completely general. We do this by studying only the extreme corners of the contact, and which we assume, for now, are both closed and adhered. The form of the contact and the loading then enter the solution only through the generalised stress intensity factors, $K_{I}$, $K_{I I}$, defining the loading on a monolithic semi-infinite wedge. So, we begin by stating Williams' solution [2] for the stress at the tip of a semi-infinite sharp notch, of included angle in the solid of $2 \alpha$, which shows that the state of stress may be written in the form

$$
\sigma_{i j}(r, \theta)=K_{I} r^{\lambda_{I}-1} f_{i j}^{I}(\theta)+K_{I I} r^{\lambda_{I I}-1} f_{i j}^{I I}(\theta),
$$

where $i, j \in\{r, \theta\}$, and, assuming plane strain, 


$$
\sigma_{\text {说 }}(r, \theta)=v\left(\sigma_{r r}(r, \theta)+\sigma_{\theta \theta}(r, \theta)\right)
$$

where $r$ is the radial distance from the notch tip, the angle $\theta$ is measured from the notch bisector and taken to be positive in the counter-clockwise direction, the eigenvalues $\lambda_{I}, \lambda_{I I}$, are the lowest roots of the equations

$$
\begin{aligned}
& \lambda_{I} \sin 2 \alpha+\sin 2 \alpha \lambda_{I}=0 \\
& \lambda_{I I} \sin 2 \alpha-\sin 2 \alpha \lambda_{I I}=0
\end{aligned}
$$

and the generalised stress intensity factors are defined along the bisector of the 'notch' $(\theta=0)$ as

$$
\begin{aligned}
& K_{I}=\lim _{r \rightarrow 0} \sigma_{\theta \theta}(r, 0) r^{1-\lambda_{I}} \\
& K_{I I}=\lim _{r \rightarrow 0} \sigma_{r \theta}(r, 0) r^{1-\lambda_{I I}}
\end{aligned}
$$

Williams' solution may be written in an alternative form that is more suitable for frictional contacts. The punch has an included angle $\phi$, and is in contact with a half-plane, as shown in Fig. 1, so that the total included angle is $2 \alpha=\phi+\pi$. The contact interface lies along the line $\theta=(\pi-\phi) / 2$, and is denoted $\theta_{i n t}$, while the distance from the notch tip along the interface line is $x$. The direct $p(x)$, and shearing $q(x)$, interfacial tractions may therefore be written as

$$
\begin{aligned}
& -p(x) \equiv \sigma_{\theta \theta}\left(x, \theta_{i n t}\right)=K_{I} x^{\lambda_{I}-1} f_{\theta \theta}^{I}\left(\theta_{i n t}\right)+K_{I I} x^{\lambda_{I I}-1} f_{\theta \theta}^{I I}\left(\theta_{i n t}\right) \equiv K_{I}^{0} x^{\lambda_{I}-1}+K_{I I}^{0} x^{\lambda_{I I}-1} \\
& q(x) \equiv \sigma_{r \theta}\left(x, \theta_{i n t}\right)=K_{I} x^{\lambda_{I}-1} f_{r \theta}^{I}\left(\theta_{i n t}\right)+K_{I I} x^{\lambda_{I I}-1} f_{r \theta}^{I I}\left(\theta_{i n t}\right) \equiv K_{I}^{0} x^{\lambda_{I}-1} g_{r \theta}^{I}+K_{I I}^{0} x^{\lambda_{I I}-1} g_{r \theta}^{I I}
\end{aligned}
$$

where compression is taken to be positive, and the generalised stress intensity factors calibrated along the interface line are denoted $K_{n}^{0}$, where $n \in\{I, I I\}$, and are found from

$$
K_{I}^{0}=K_{I} f_{\theta \theta}^{I}\left(\theta_{i n t}\right), \quad K_{I I}^{0}=K_{I I} f_{\theta \theta}^{I I}\left(\theta_{i n t}\right)
$$

where

$$
g_{r \theta}^{I}=\frac{f_{r \theta}^{I}\left(\theta_{i n t}\right)}{f_{\theta \theta}^{I}\left(\theta_{i n t}\right)}, \quad g_{r \theta}^{I I}=\frac{f_{r \theta}^{I I}\left(\theta_{i n t}\right)}{f_{\theta \theta}^{I I}\left(\theta_{i n t}\right)}
$$

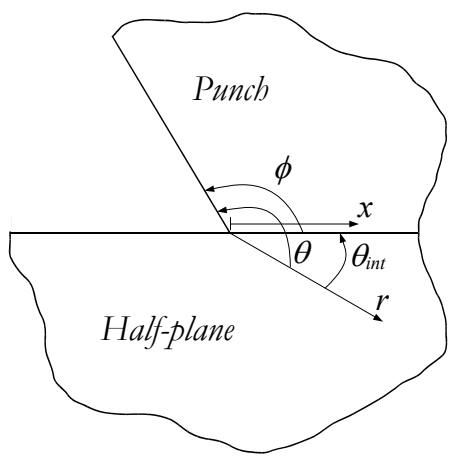

Figure 1: A diagram of the idealised geometry considered, including the coordinate system.

\section{EDGE SEPARATION}

illiams' solution displays a power law variation of the stress field with radial distance from the notch tip and expresses this as a series expansion, of which we consider only the first two terms; $K_{I}$ and $K_{I I}$. Because each term in the series is raised to a different power (except in the case of an edge crack when $\phi=\pi$ ), the relative 
strengths of the two competing terms vary with radial distance from the notch tip, which creates an intrinsic length scale in the solution; the mode $I$ term is more strongly singular and dictates contact edge behaviour, whilst the mode $I I$ term less strongly singular (or for punch angles less than $77.4^{\circ}$ the mode II term is bounded) and controls the stress field slightly further from the sharp corner. This length scale can be emphasised by replacing the two parameters $K_{I}$ and $K_{I I}$ in Williams' solution with two new composite parameters $d_{0}$ and $G_{0}$. The definition of these quantities involves raising the generalised stress intensity factors $K_{I}$ and $K_{I I}$, to fractional powers, and for this reason, we modify the definition depending on whether the remote loads excite positive or negative values of $K_{I}$ and $K_{I I}$, in order to avoid imaginary solutions. For simplicity, let us first consider the case when the remote loads excite positive values of the generalised stress intensity factors $K_{I}$ and $K_{I I}$. In this case, $d_{0}$ and $G_{0}$ are defined as

$$
d_{0}=\left[\frac{K_{I I}}{K_{I}}\right]^{\frac{1}{\lambda_{I}-\lambda_{I I}}}, \quad G_{0}=K_{I}^{\frac{\lambda_{I I}-1}{\lambda_{I I}-\lambda_{I}}} K_{I I}^{\frac{\lambda_{I}-1}{\lambda_{I}-\lambda_{I I}}}
$$

where it is now clear that $d_{0}$ has the physical significance of representing the boundary, in some way, between mode $I$ domination and mode II domination of the stress field, whilst $G_{0}$ represents the magnitude of loading. Eq. (1) can now be re-written as

$$
\frac{\sigma_{i j}(r, \theta)}{G_{0}}=\left(\frac{r}{d_{0}}\right)^{\lambda_{I}-1} f_{i j}^{I}(\theta)+\left(\frac{r}{d_{0}}\right)^{\lambda_{I I}-1} f_{i j}^{I I}(\theta) .
$$

We now, as in Eq. (7) and (8), write out the direct $p(x)$, and shearing $q(x)$, tractions, but this time with the alternative formulation of the stress field, and we also, for compactness, use the shorthand $f_{i j}^{n} \equiv f_{i j}^{n}\left(\theta_{i n t}\right)$, as

$$
\begin{aligned}
& \frac{-p(x)}{G_{0}} \equiv \frac{\sigma_{\theta \theta}\left(r, \theta_{i n t}\right)}{G_{0}}=\left(\frac{x}{d_{0}}\right)^{\lambda_{I}-1} f_{\theta \theta}^{I}+\left(\frac{x}{d_{0}}\right)^{\lambda_{I I}-1} f_{\theta \theta}^{I I} \\
& \frac{q(x)}{G_{0}} \equiv \frac{\sigma_{r \theta}\left(r, \theta_{i n t}\right)}{G_{0}}=\left(\frac{x}{d_{0}}\right)^{\lambda_{I}-1} f_{r \theta}^{I}+\left(\frac{x}{d_{0}}\right)^{\lambda_{I I}-1} f_{r \theta}^{I I} .
\end{aligned}
$$

When the remote loads are such that the generalised stress intensity factors $K_{I}$ and $K_{I I}$, are of the same sign (either both positive or both negative), Williams' solution implies that the direct traction $p(x)$, is of a different sign in the mode $I$ and mode $I I$ dominated regions of the stress field. When $K_{I}$ and $K_{I I}$ are both positive, there is implied separation at the edge of contact, but closure is implied further away from the contact edge. Conversely, when $K_{I}$ and $K_{I I}$ are both negative, closure is implied at the edge of contact, but separation is implied to extend from the interior. It is particularly important in the latter case, to appreciate that a state of separation may not actually arise because, by the time the mode II solution dominates the mode I solution, the next term in the series, which has not been found, may be important. In any case, when the generalised stress intensity factors take on similar signs, the length along the interface at which Williams' solution implies that the boundary between separation and closure lies, based on violations of the condition $p(x)>0$, is denoted $x_{0}$, and is given simply by setting $p(x)=0$, and solving for the value of $x / d_{0}$ at which this condition is met, which gives

$$
\frac{x_{0}}{d_{0}}=\left[-\frac{f_{\theta \theta}^{I I}}{f_{\theta \theta}^{I}}\right]^{\frac{1}{\lambda_{I}-\lambda_{I I}}}
$$

Note that, perhaps surprisingly, when represented in this way, the strength of the remote loading $G_{0}$, does not influence the position of the boundary between separation and closure $x_{0}$, if normalised by $d_{0}$. Also, note that, for remote loading that results in $K_{I}$ and $K_{I I}$ taking on opposing signs, Williams' solution implies no change in sign of the direct traction 
$p(x)$. Thus, when $K_{I}$ is negative and $K_{I I}$ is positive, closure is implied in both mode $I$ and mode $I I$ regions. Conversely, when $K_{I}$ is positive and $K_{I I}$ is negative, Williams' solution implies separation throughout the whole of the region controlled by the asymptote. This implication of gross separation means that Williams' solution cannot yield any further characterisation of contact edge behaviour, therefore we do not consider this case in the subsequent analysis.

\section{REGIONS OF FRICTIONAL SLIP}

o estimate the implied extent of slip, based on violations of the slip condition, the adhered interfacial tractions must be substituted into the slip condition $q(x)= \pm \not p(x)$, where $f$ is the coefficient of friction. This calculation reveals the position of all the implied boundaries between stick and slip within the edge region controlled by the asymptote, where we denote the distance from the corner to any point at which slip condition is just met as $x_{s}$. For simplicity, we begin by considering the case when $K_{I}$ and $K_{I I}$ are both positive, such that a small region of edge separation is implied, with an adjacent slip region. Explicitly, the slip extent, $x / d_{0}$, is given by

$$
\frac{x_{s}}{d_{0}}=\left[-\frac{f_{r \theta}^{I I} \pm f \cdot f_{\theta \theta}^{I I}}{f_{r \theta}^{I} \pm f \cdot f_{\theta \theta}^{I}}\right]^{\frac{1}{\lambda_{1}-\lambda_{I I}}}
$$

Plots of the implied slip extent (from Eq. (16)) as well as a line showing the implied separated region (from Eq. (15)), for the case when $K_{I}$ and $K_{I I}$ are both positive, are shown in Fig. 2, for three sample punch angles $\phi=\left\{60^{\circ}, 90^{\circ}, 120^{\circ}\right\}$.
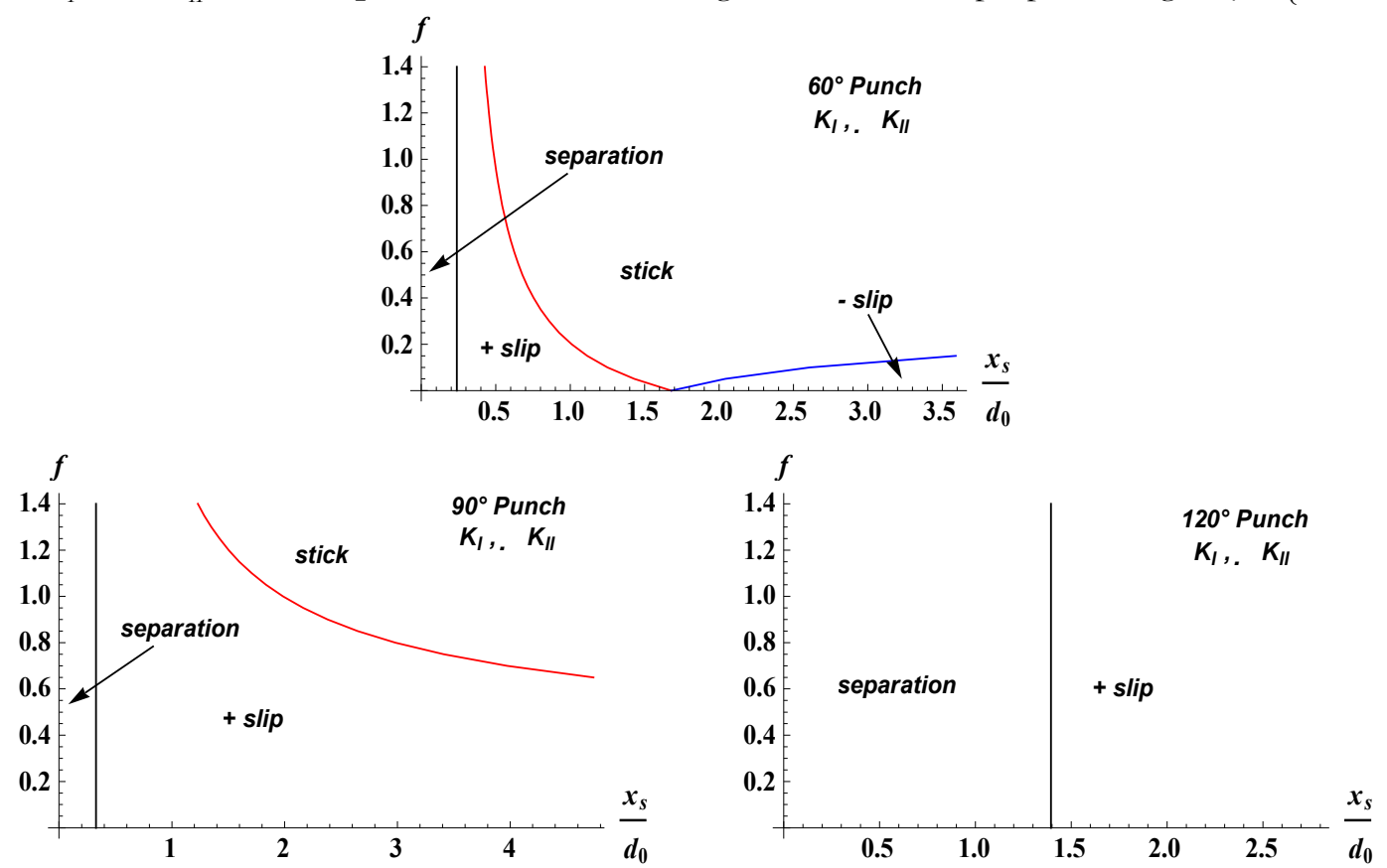

Figure 2: Plots of the implied regions of slip, stick, and separation, when both $K_{I}$ and $K_{I I}$ are positive, for punch angles of $\phi=\left\{60^{\circ}, 90^{\circ}, 120^{\circ}\right\}$, where the black line denotes the boundary between closure and separation, the red line the position at which the $q(x)=+\not p(x)$ condition is met, and the blue line the position at which the $q(x)=-\not p(x)$ condition is met.

For the case when the remote loads excite stresses that result in a negative $K_{I}$ and a positive $K_{I I}$, the parameters $d_{0}$ and $G_{0}$ must be defined differently from Eq. (11), to avoid raising a negative number to a fractional power. Thus, we define a new parameter $\bar{K}_{n}=-K_{n}$, and substitute it in place of the negative stress intensity factor, so that, for example when $K_{I}$ is negative and $K_{I I}$ is positive we have 


$$
d_{0}=\left[\frac{K_{I I}}{\bar{K}_{I}}\right]^{\frac{1}{\lambda_{I}-\lambda_{I I}}}, \quad G_{0}=\bar{K}_{I}^{\frac{\lambda_{I I}-1}{\lambda_{I I}-\lambda_{I}}} K_{I I}^{\frac{\lambda_{I}-1}{\lambda_{I}-\lambda_{I I}}}
$$

For this case, the stresses, instead of being given by Eq. (12), are instead given by

$$
\frac{\sigma_{i j}(r, \theta)}{G_{0}}=-\left(\frac{r}{d_{0}}\right)^{\lambda_{I}-1} f_{i j}^{I}(\theta)+\left(\frac{r}{d_{0}}\right)^{\lambda_{I I}-1} f_{i j}^{I I}(\theta)
$$

so that the direct $p(x)$, and shearing $q(x)$, are given by

$$
\begin{aligned}
& \frac{-p(x)}{G_{0}} \equiv \frac{\sigma_{\theta \theta}\left(r, \theta_{i n t}\right)}{G_{0}}=-\left(\frac{x}{d_{0}}\right)^{\lambda_{I}-1} f_{\theta \theta}^{I}+\left(\frac{x}{d_{0}}\right)^{\lambda_{I I}-1} f_{\theta \theta}^{I I} \\
& \frac{q(x)}{G_{0}} \equiv \frac{\sigma_{r \theta}\left(r, \theta_{i n t}\right)}{G_{0}}=-\left(\frac{x}{d_{0}}\right)^{\lambda_{I}-1} f_{r \theta}^{I}+\left(\frac{x}{d_{0}}\right)^{\lambda_{I I}-1} f_{r \theta}^{I I}
\end{aligned}
$$

When $K_{I}$ is negative and $K_{I I}$ is positive, closure is implied through the asymptotic region. However, depending on the punch angle, $\phi$, and the coefficient of friction, $f$, various slip regions are implied at the edge and/or interior of the contact. To compute the implied slip extents we substitute Eq. (19) and (20) into the slip condition $q(x)= \pm f p(x)$, and solve for $x / d_{0}$, again, denoting any boundary between stick and slip as $\chi_{s}$, which gives

$$
\frac{x_{s}}{d_{0}}=\left[\frac{f_{r \theta}^{I I} \pm f \cdot f_{\theta \theta}^{I I}}{f_{r \theta}^{I} \pm f \cdot f_{\theta \theta}^{I}}\right]^{\frac{1}{\lambda_{I}-\lambda_{I I}}}
$$
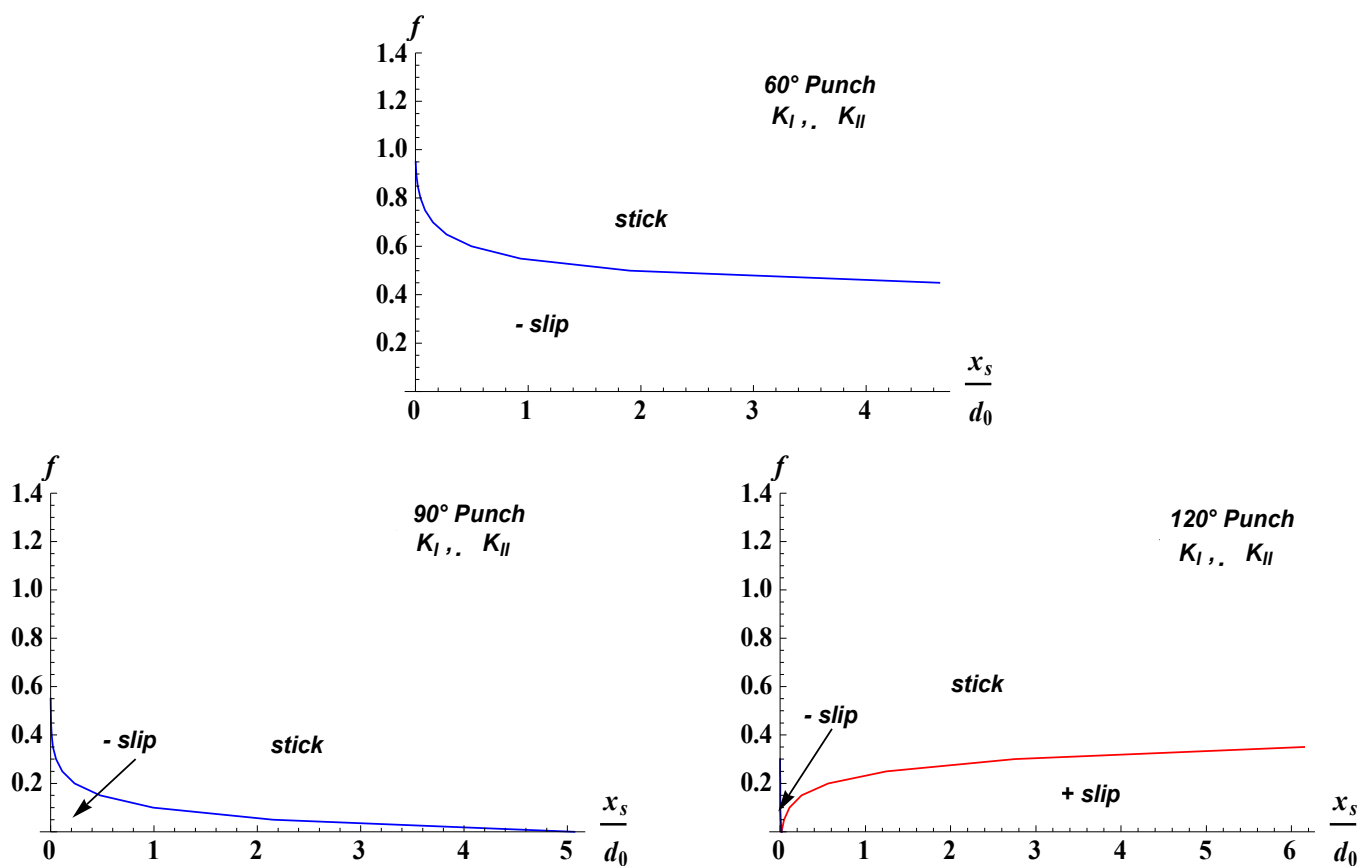

Figure 3: Plots of the implied regions of slip and stick, when $K_{I}$ is negative and $K_{I I}$ is positive, for punch angles of $\phi=\left\{60^{\circ}, 90^{\circ}, 120^{\circ}\right\}$, where the red line denotes the position at which the $q(x)=+\not p(x)$ condition is met, and the blue line the position at which the $q(x)=-f p(x)$ condition is met. 
Plots of the implied slip regions (from Eq. (21)) as a function of the coefficient of friction $f$, for the case when $K_{I}$ is negative and $K_{I I}$ is positive, are shown in Fig. 3, for three sample punch angles $\phi=\left\{60^{\circ}, 90^{\circ}, 120^{\circ}\right\}$. Finally, for cases in which both $K_{I}$ and $K_{I I}$ are negative, separation is implied to extend from the interior of the contact, with closure and a slip region at the edge of contact whose extent is controlled by the next term in a series expansion. We do not consider this possibility here or in any subsequent analysis.

\section{The Plastic Zone}

$\mathrm{T}$

he implied elastic state of stress near a sharp feature is singular, but in practice the singular behaviour is truncated by the presence of a plastic zone incorporating a process region, and whose extent is determined by the strength of the applied load and the yield strength of the material. In order to determine the size of the plastic zone, implied by violations of the yield condition, we use the second invariant of deviatoric stress

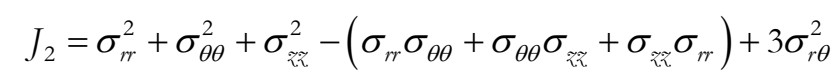

and note that, with this scaling von Mises yield condition is

$$
J_{2}=3 k^{2}
$$

where $k$ is the yield stress of the material in pure shear. We then modify Eq. (12) to be specifically along the interface of the contact, i.e.

$$
\frac{\sigma_{i j}\left(x, \theta_{i n t}\right)}{G_{0}}=\left(\frac{x}{d_{0}}\right)^{\lambda_{1}-1} f_{i j}^{I}\left(\theta_{i n t}\right)+\left(\frac{x}{d_{0}}\right)^{\lambda_{I I}-1} f_{i j}^{I I}\left(\theta_{i n t}\right)
$$

and use this expression (Eq. (24)) together with Eq. (22), and then simplify the result, to give

$$
J_{2}=G_{0}^{2}\left\{\left(\frac{x}{d_{0}}\right)^{2\left(\lambda_{I}-1\right)} p_{I}\left(\theta_{i n t}\right)+\left(\frac{x}{d_{0}}\right)^{2\left(\lambda_{I I}-1\right)} p_{I I}\left(\theta_{i n t}\right)+\left(\frac{x}{d_{0}}\right)^{\left(\lambda_{I}+\lambda_{I I}-2\right)} p_{I-I I}\left(\theta_{i n t}\right)\right\}
$$

where $p_{I}\left(\theta_{i n t}\right), p_{I I}\left(\theta_{i n t}\right)$, and $p_{I-I I}\left(\theta_{i n t}\right)$ represent the $\theta$-dependence of the mode $I$, mode $I I$, and mixed mode terms in the solution, respectively. We now impose the yield condition given in Eq. (23), denote the size of the plastic zone along the interface line $x_{p}$, and solve for $G_{0} / k$, which gives

$$
\frac{G_{0}}{k}=\sqrt{3}\left\{\left(\frac{x_{p}}{d_{0}}\right)^{2\left(\lambda_{I}-1\right)} p_{I}\left(\theta_{i n t}\right)+\left(\frac{x_{p}}{d_{0}}\right)^{2\left(\lambda_{I I}-1\right)} p_{I I}\left(\theta_{i n t}\right)+\left(\frac{x_{p}}{d_{0}}\right)^{\left(\lambda_{I}+\lambda_{I I}-2\right)} p_{I-I I}\left(\theta_{i n t}\right)\right\}^{-\frac{1}{2}}
$$

If the punch angle $\phi$, is specified, Eq. (25) can be used to determine the size of the plastic zone $x_{p} / d_{0}$, as a function of strength of the applied load $G_{0} / k$, and this is plotted for punch angles of $\phi=\left\{60^{\circ}, 90^{\circ}, 120^{\circ}\right\}$ in Fig. 4. This figure illustrates the perhaps surprising property that the relationship between the strength of the applied load $G_{0} / k$, and the normalised size of the plastic zone specified along the interface line $x_{p} / d_{0}$, is not monotonically increasing. This is because, as described in Hills et al. [1], as strength of the remote load is increased, the plastic front rotates and changes from being very mode $I$ like when loaded lightly to being very mode II like in character when loaded heavily. Therefore, although the maximum radius of the plastic zone does increase monotonically in size with stronger loading, when specified along the interface line, the plastic radius takes on its maximum value when the remote loading causes the maximum plastic radius to coincide with the interface line. The plastic length along the interface then decreases for stronger loads, because the maximum plastic radius rotates away from the line corresponding to the frictional interface. 


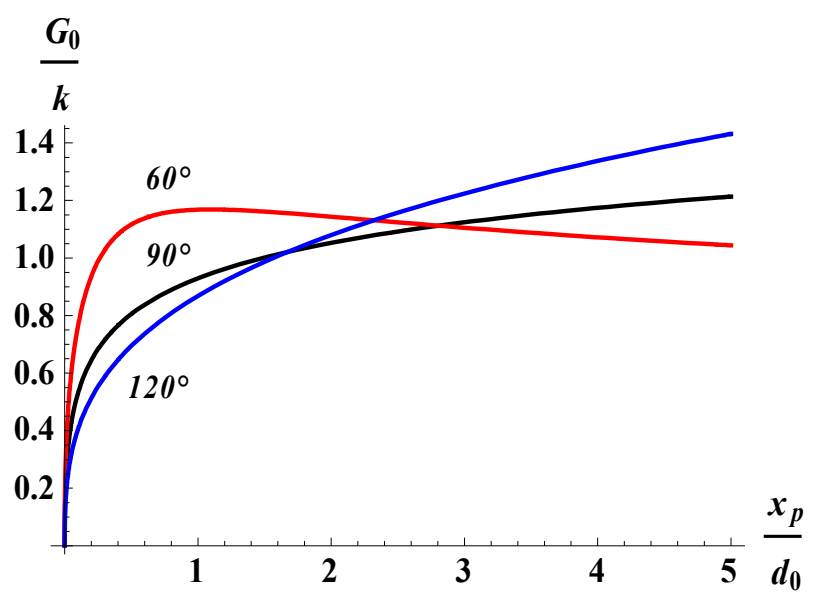

Figure 4: A plot showing the size of the plastic zone $\mathrm{x}_{\mathrm{p}} / \mathrm{d}_{0}$, as a function of strength of the applied load $\mathrm{G}_{0} / \mathrm{k}$, for example punch angles of $\phi=\left\{60^{\circ}, 90^{\circ}, 120^{\circ}\right\}$, shown in red, black, and blue, respectively.

\section{Determination of the Dominant Slip Process: Plastic vs. Frictional}

$\mathrm{W}$ e have described how to calculate the relative sizes of the implied regions of adhesion, slip, and separation, and also how the size of the plastic zone specified along the interface line scales with remote load, so we are in a position to address the question that motivated this analysis; "when is the frictional slip enveloped by plastic slip, so that the corner is effectively a notch in a monolithic material?" The first thing to be said on this issue is that, if frictional slip is implied to extend out past the mode II region of the solution and into the region where bounded terms control the behaviour of the contact, then whether or not the plastic zone fully envelops the zone of frictional slip at the edge of contact cannot be determined through examination of the implications Williams' asymptotic solution. To find out when this condition obtains we consider the value of the implied traction ratio in the mode II region, which, for the example punch angles of $\phi=\left\{60^{\circ}, 90^{\circ}, 120^{\circ}\right\}$, is $g_{r \theta}^{I I}=\{0.3224,-0.2189,-0.8500\}$, respectively. Thus, if the coefficient of friction is less than the absolute value of this implied traction ratio, i.e. if $f<\left|g_{r} I\right|$, then the question of whether or not the frictional slip zone is enveloped by plasticity cannot be determined by consideration of Williams' asymptote. If, however, slip is not implied to extend past the mode II region of the solution, and is contained within the asymptote, i.e. if $f \geq\left|g_{r}^{I I}\right|$, then further examination of the implications of Williams' solution is merited. For cases when $f \geq\left|g_{r} I\right|$ and thus adhesion is implied in the mode II region of the solution, we can calculate the strength of load required to imply a plastic zone of equal size to the implied zone of frictional slip. This is achieved by setting $\left(x_{p} / d_{0}\right)=\left(x_{s} / d_{0}\right)$ in Eq. (25), and solving for the value of $G_{0} / k$. The result of this calculation is plotted against the coefficient of friction $f$, for punch angles of $\phi=\left\{60^{\circ}, 90^{\circ}, 120^{\circ}\right\}$, in Fig. 5. Also plotted in Fig. 5, are three horizontal dashed lines showing the values of $\left|g_{r \theta}^{I I}\right|$ for the three punch angles considered. This information is added because, as stated above, if the coefficient of friction is less than this value, i.e. if $f<\left|g_{r \theta}^{I I}\right|$, then slip is implied to extend outside the region controlled by the asymptote, and the magnitude of load required to imply a process zone of equal size to the frictional slip zone cannot be calculated within the asymptote. The solid lines in Fig. 5 plot the magnitude of load $G_{0} / k$, that results in the conditions $\left(x_{p} / d_{0}\right)=\left(x_{s} / d_{0}\right)$ and $f \geq\left|g_{r \theta}^{I I}\right|$ both being met, while the dotted lines plot the value of $G_{0} / k$ that result in only the first condition being satisfied. So, for loads greater in strength than the solid line in Fig. 5, slip is implied only in the mode $I$ dominated region (because $f>\left|g_{r}^{I I}\right|$ ), and the process zone is implied to be larger than the slip zone in this region. Thus, in this case the contact is expected to behave like a notch. For loads greater than the dotted lines in Fig. 5, which 
are in the range $f<\left|g_{r \theta}^{I I}\right|$, the process zone is implied to be as large as the slip zone in the mode $I$ dominated region, but a second slip zone is implied to extend past the mode II dominated region. These dotted lines are included in the figure to distinguish between cases in which the slip zone in the mode $I$ dominated region is fully enveloped by plasticity at a coefficient of friction below $\left|g_{r \theta}^{I I}\right|$, which implies that for coefficients of friction greater than $\left|g_{r \theta}^{I I}\right|$ there will be no slip, from the cases in which the magnitude of load required to envelop the slip zone in the mode $I$ dominated region does not appear on the plot, which implies that, for the range of load shown in the figure, the slip zone is larger than the process zone and that the contact will slip. The curves in Fig. 5 corresponding to the case $\phi=120^{\circ}$ (shown in blue) illustrate these two distinct possibilities; when $\mathrm{K}_{\mathrm{I}}$ and $\mathrm{K}_{\mathrm{II}}$ are both positive (Left) neither dotted nor solid lines appear on the plot and the slip zone is implied to be larger than the plastic zone in the range of $f$ and $G_{0} / k$ shown in the figure, but when $K_{I}$ is negative and $K_{I I}$ is positive (Right) a dotted line appears on the plot and thus if $f \geq \mid g_{r \theta}$ II $\mid$ no slip is implied.
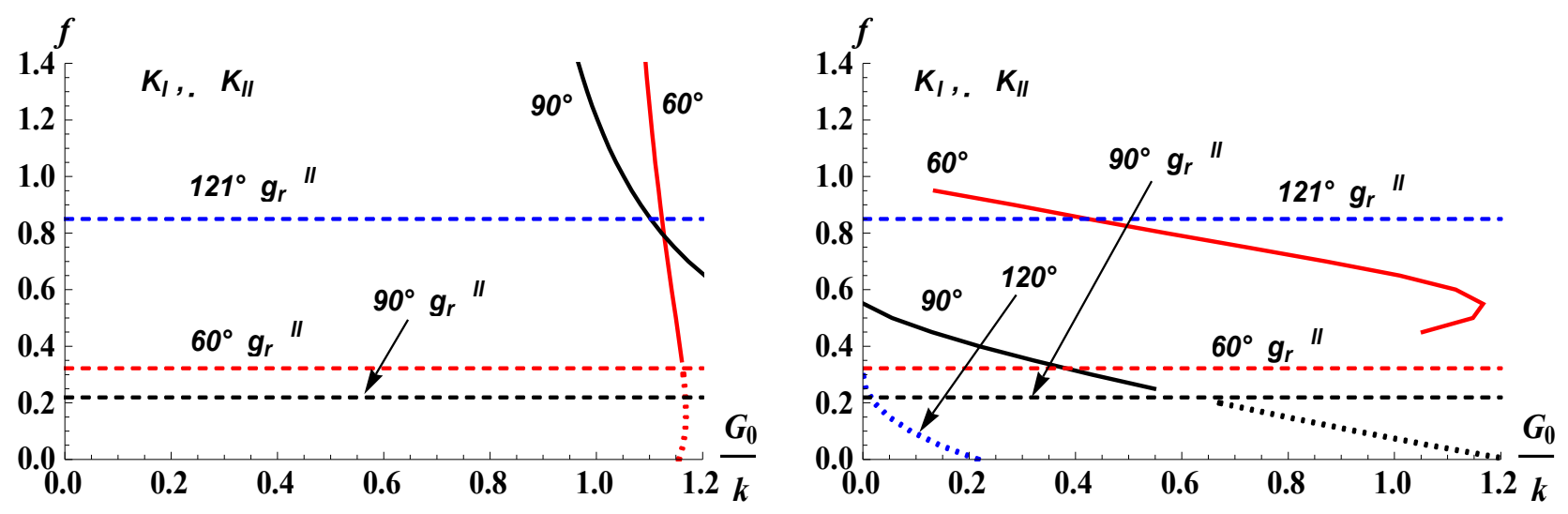

Figure 5: Plots showing the strength of load $G_{0} / k$, above which the process zone and frictional slip zone at the edge of contact are implied to be of equal size when $f>\left|g_{r \theta}^{I I}\right|$ (solid lines), and when $f<\left|g_{r \theta}^{I I}\right|$ (dotted lines), for punch angles of $\phi=\left\{60^{\circ}, 90^{\circ}, 120^{\circ}\right\}$, plotted in red, black, blue, respectively. This is done for the case when both $K_{I}$ and $K_{I I}$ are positive (Left) and when $K_{I}$ is negative and $K_{I I}$ is positive (Right). Also shown are the values of $\left|g_{r \theta}^{I I}\right|$ for each punch angle (horizontal dotted lines), using the same colour scheme as for the other lines.

\section{CONCLUSIONS}

$\mathrm{T}$ his paper develops further our physical understanding of the physical implications of the asymptotic form given by Williams, allowing not only mode mixity but also the competing effects of frictional and plastic slip to be treated; the former, but not the latter, is independent of the magnitude of the load. The 'output' of this paper is therefore a specific answer to the question 'When is a sharp contact corner rigorously notch-like?' and equally 'When is frictional slip likely to be important?'. Space limitations preclude the application of the results to real problems, which requires calibrations for $\mathrm{K}_{\mathrm{I}}$ and $\mathrm{K}_{\mathrm{II}}$ in terms of the applied loads. This last step then permits these results to be applied directly to any complete fretting (or potentially fretting) contact, by simply 'pasting in' this asymptotic form.

\section{ACKNOWLEDGEMENTS}




\section{REFERENCES}

[1] Hills, D.A., Dini, D., Characteristics of the Process Zone at Sharp Notch Roots, Int. J. Solid Struct., 48 (2011), $2177-$ 2183.

[2] Williams, M. L., Stress singularities resulting from various boundary conditions in angular plates in extension. J. Appl. Mech., 19 (1952), 526-528. 